\title{
WMSN: ANALYSIS OF AGRICULTURAL SOIL ENVIRONMENT MONITORING USING WIRELESS MULTIMEDIA SENSOR NETWORK
}

\author{
E.Manjula, Pachaiyappas college, India \\ S.Djodiltachoumy, Pachaiyappas college, India \\ manjulababu123@gmail.com \\ djodilatchoumy@hotmail.com
}

\begin{abstract}
This paper proposes an environmental monitoring for agriculture soil using Wireless Multimedia Sensor Network. WMSN is used to monitor the agriculture soil environment. In order to ensure optimal use of the sensor nodes in WMSN and accurate access to the collected data, the routing protocols with both energy efficiency and quality of service ( QoS) properties are necessary for the transmission of photo, video and scalar data. This paper proposes a priority aware energy efficient QoS based data transmission for agriculture soil environment and also detects the pest. It utilizes the available bandwidth and data rate in the best way and in order to be more energy efficient during data transmission. For performance analysis, the proposed system is simulated using Java and the results of the experiments suggest that the proposed algorithm is more efficient compared to other methods in terms of delay and energy consumption. The sink node provides the solution based on these collected values.
\end{abstract}

Keywords: WMSN, Wireless Sensor Network, QoS, Routing Protocols

\section{INTRODUCTION}

Wireless networks for sensors have emerged as a promising technology for embedded systems. Lowcost, low-power, multi-functional, fully integrated digital electronics are becoming more WSNs, and wireless networking is proliferating. It typically consists of a large number of intelligent, batterypowered sensor nodes with sensing, processing, and wireless communication capabilities. The sensor sends collected scalar data via radio transmitter, either directly or via multiple wireless hops to a sink. Real time objects tracking, environmental and healthcare monitoring are the widely used

Copyright $(\subset$ Authors applications in WSN [1]. Sensor node is designed for sensing scalar data and most of the applications have delay and fault tolerant with high energy consumption.

A priority aware energy efficient QoS based data transmission with pest detection is proposed for reducing energy consumption and increasing crop yield production. The foremost hand-outs of this study are being summarized as: (1) To design a costeffective and energy-effective network of intelligent wireless media sensors for monitoring the soil environment; (2) To develop a sleep wake up scheduling for energy efficient data collection; (3) To create a priority aware QoS based data transmission; (4) To increase the crop yield production, periodically check the soil fertility; (5) To detect the insects and pest affected leafs in the agricultural soil.

The remaining part of this paper is arranged as follows. Section2 reviews the associated work of monitoring the environment and the transfer of data. In section 3, the network model and notations are implemented. The proposed priority-based, energyefficient QoS-based data transfer and insect detection are defined in Section 4. Section 5 assesses success by experimental outcomes, and this article is eventually concluded in section 6 .

\section{RELATED WORK}

\subsection{Environment Monitoring}

Mao et al.[8] present CitySee, an urban-area realtime $\mathrm{CO} 2$ monitoring system using sensor networks, structures the remainder of this article as follows. Large-scale wireless sensor networks comprising 1096 relay nodes and 100 sensor nodes are being used for simulation in "Wuxi \& "China et al.[9]" 
analysed the huge resolved Ultrafine Particles (UFPs) data set in Zurich, Switzerland, for measurements of air quality. Those that threaten human health, "such as nitrogen dioxide ( $\mathrm{NO} 2$ ), carbon monoxide $(\mathrm{CO})$, ozone $(\mathrm{O} 3)$ and ultrafine particles (UFPs)", are the target pollutants. Severe conditions such as respiratory illnesses, cardiovascular complications, etc. are responsible for these toxins.

Jiang et al.,[10] examine the realistic experience of introducing citizen recognition for urban environmental monitoring. A bottom-up model is defined and evaluated in which people create and use sensors for monitoring the environment. It focuses on a case study using NO2 sensors for air quality monitoring from the Amsterdam Smart Citizens Lab. The implementation of a distributed monitoring system in a commercial greenhouse using the WSN was presented in[11]. Initially, a WSN prototype was developed to analyse the effects of environmental factors on the efficiency of the network's operation and to evaluate its performance and the viability of its operation in a commercial greenhouse.

Liu[12] in the IoT greenhouse, developed an intelligent environmental monitoring system for precise grape cultivation. Sensors were designed to gain numerous types of data that were then combined and stored, processed and modified at the gateway in the background. Environmental variables were air temperature, air moisture, illumination, soil moisture, and $\mathrm{CO} 2$ concentration. In addition, videos and photographs were taken during the production of grapes.

Air Quality Monitoring was presented in [13] using a LEACH-based aggregation of data. The author develops an efficient aggregation technique that is acceptable for the environment of air pollution, since few key meteorological factors such as wind speed and atmospheric turbulence affect air quality.

\subsection{WMSN in Agriculture}

Shi et al., [14] present a robust multi-support and scalable Wireless Multimedia Sensor Network (WMSN) platform for Environmental Event Detection. Camera is used for the identifying disease-infected plants, the inexpensive paired system (CCD). A context-aware multi-agent-based

Copyright (C) Authors knowledge collection using WMSN for agriculture monitoring is proposed in [15]. There are three types of circumstances that are taken into account, such as crisis detection, chronological and computational history to classify diseased plants, weeds, fires, and soil fertility analysis based on soil parameters. $B_{i}$ [16] develops an intelligent multimedia sensor network monitoring architecture and links different sensors to the monitoring network to monitor crop areas for one month It measures the ambient temperature and humidity of the crop area, the light intensity and carbon dioxide concentration and transmits the measurement data to the user.

Indumathi et al. ,[17] introduce an intelligent method of detection of WMSN plant disease. A novel Disease Detection Method (DDS) is proposed to detect and classify diseases in leaves. The statistical-based thresholding technique is suggested for segmentation that is less complex compared to $\mathrm{k}$ means clustering methodology. The characteristics extracted from the segmented image are transmitted via sensor nodes to the monitoring site, where the analysis and classification is carried out using the classifier Support Vector Machine.

Libo et al.,[18] produce a rape disease diagnostic technique that uses WMSN. This technique will make the first decision on the plant vegetation, knowing how to send photos to the control centre to continue exploring, to move forward.

\subsection{Data Transmission in WMSN}

In order to improve the transmission efficiency of images compressed by embedded wavelet transform zerotrees, Bouchemel et al.[19] propose an energyefficient low-complexity scheme to define partitioning in hierarchical tree algorithms in WMSNs based on a multiplexing technique of division of orthogonal frequency. In this method, a simple post-inverse discrete Fourier transform modified $\mu$ nonlinear transformation, known as $\mu$ MNLT, is used.

A new evolutionary-game-based routing (EGR) protocol for WMSNs is suggested in[20] with several sinks, in which the theory of evolutionary games is used to select CHs. In light of the covering field of perspectives on interactive media sensor hubs, a calculation to diminish information repetition is additionally given in EGR. This 
calculation diminishes the quantity of repetitive transmissions, consequently expanding energy utilization and organization power.

Hasan et al.[21] suggest a statistical model for the routing-determination process of a novel quality-of - service (QoS). This framework allows for a wide variety of real-time intensive media to decide the optimal route to provide adequate shared radio that meets the QoS. In order to control adaptive switching of hop-by - hop QoS routing protocols, the mathematical model is based on the Lagrangian relaxation approach.
Genta et al.[7] are proposing a routing algorithm combining dynamic cluster formation, cluster head selection and multipath routing formation for data communication to reduce energy consumption as well as overhead routing. The algorithm uses a genetic algorithm (GA)-based meta-heuristic optimization to dynamically choose the best path based on the function of minimum distance cost and least energy dissipation.

\section{PROPOSED NETWORK MODEL}

The proposed network model and notation used in the paper are defined in this section. In Figure 1, the model of network is shown.

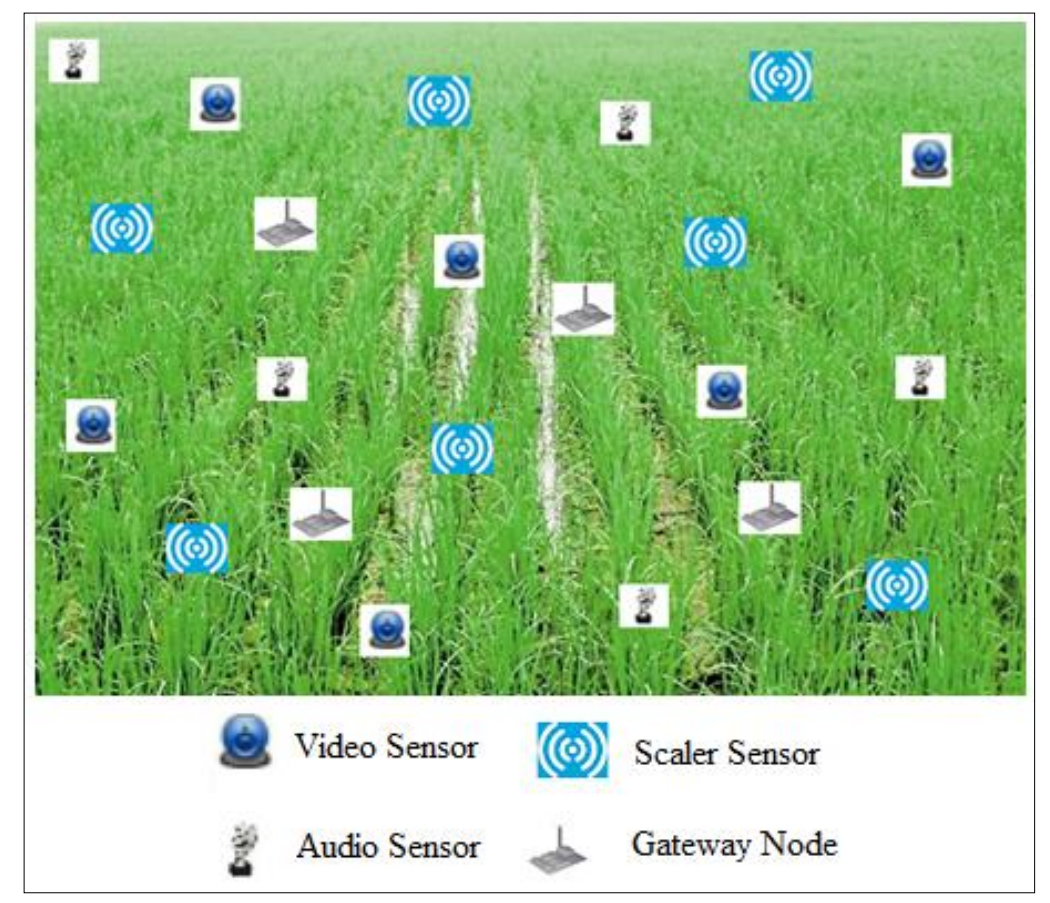

Figure 1 Model of Network

The set of connections (network), includes the number ' $n$ ' of senor multimedia nodes with the number ' $\mathrm{m}$ ' of gateway nodes. The device is generated as an undirected weighted graph denoted by $\mathrm{G}=(\mathrm{V}, \mathrm{E}, \mathrm{W})$, where $\mathrm{V}$ is a set of wireless multimedia sensor nodes (video, audio and scalar), $\mathrm{E}$ is a set of bidirectional connections, and $\mathrm{W}$ is a set of weights (distance between nodes) on the connections,. Nodes $\mathrm{u}$ and $\mathrm{v}$ are only connected when the node $u(v)$ is inside the node $v(u)$ communication range.
The sensor nodes are deployed randomly to monitor and detect in a square sensing field, and to collect information on dynamic events. There are three types of sensor node are placed video, audio and scalar sensor. The video sensor captures the video of current location and sends still images to the gateway node. The audio sensor senses the sound information like animals, birds and insects. The scalar sensor senses the soil information like moisture, temperature, nutrients. Table 1 describes the notations and mathematical symbols. 
Table 1 Symbols and Description

\begin{tabular}{|l|l|}
\hline Symbols & Description \\
\hline $\mathrm{N}$ & Total no of sensor nodes \\
\hline $\mathrm{M}$ & Total no of gateway nodes \\
\hline $\mathrm{sn}_{\mathrm{i}}$ & $\mathrm{i}^{\text {th }}$ sensor node in network \\
\hline $\mathrm{gw}_{\mathrm{i}}$ & $\mathrm{i}^{\text {th }}$ gateway node in network \\
\hline $\mathrm{e}_{\mathrm{ij}}$ & when there is a link between node $\mathrm{i}$ and $\mathrm{j}$ is $\mathrm{e}_{\mathrm{ij}}=1$ otherwise $\mathrm{e}_{\mathrm{ij}}=0$ \\
\hline dist $_{\mathrm{ij}}$ & The distance between node $\mathrm{i}$ and $\mathrm{j}$ \\
\hline neigh $_{\mathrm{i}}$ & Neighboring node list of $\mathrm{i}$ node \\
\hline engr $_{\mathrm{i}}$ & Current Energy of node $\mathrm{i}$ \\
\hline event $_{\mathrm{i}}$ & Sensed event of node $\mathrm{i}$ \\
\hline qos $_{\mathrm{i}}$ & QoS parameters for node $\mathrm{i}$ \\
\hline event $_{\mathrm{pri}}$ & Priority value for an event \\
\hline
\end{tabular}

- The sensor hubs are battery-fueled and it is extremely hard to reach and disengage the sensor batteries. To expand the life of the organization, the energy devoured by every sensor hub ought to be decreased. Rest wake planning with need cognizant QoSbased compelling information transmission to determine this issue. Every sensor have distinctive energy level based on their sorts.

- Sensors have the knowledge of their own location.
- The gateway node is responsible for transmitting the collected information to the sink.

- There is a base station on the outside of the sensing field.

- $\quad$ Base Station has knowledge of all nodes in the sensor and gateway.

\section{DESCRIPTION OF RESEARCH WORK}

This section presents the proposed priority for energy-efficient wireless multimedia sensor network data transmission in the agricultural domain, based on QoS.

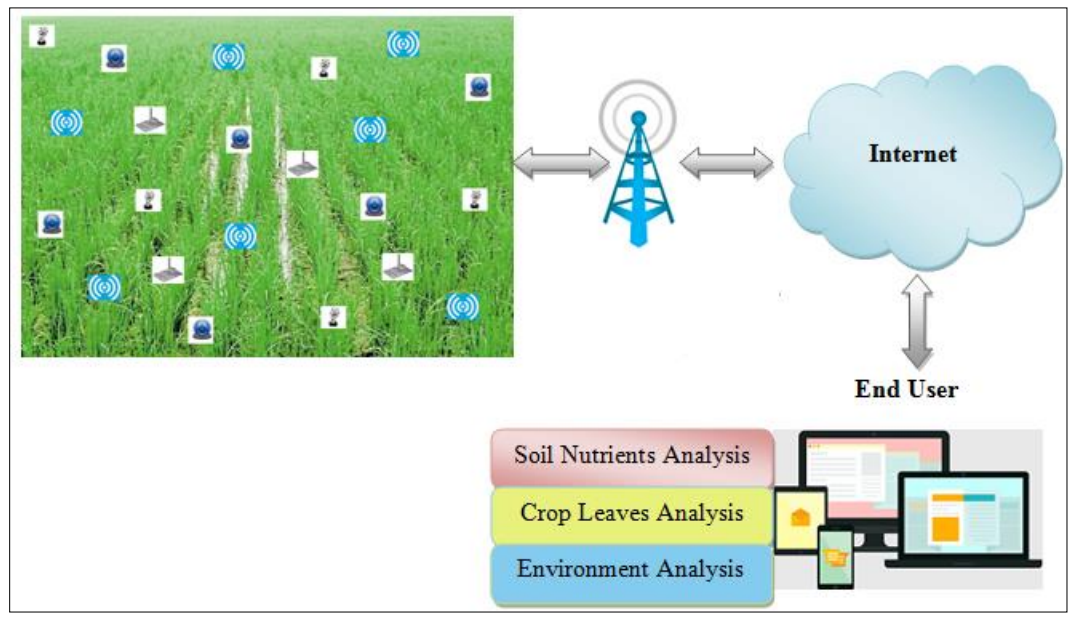

Figure 2 General Architecture

The general architecture of the work proposed is shown in Figure 2. The system model contains sensor nodes and gateway nodes deployed in agriculture area, Base Station, Internet Connection and End User Application.

Copyright (C) Authors

\subsection{System Entities}

Sensor Nodes: Sensor node is responsible to collect the information about agricultural soil. There are three types of heterogeneous sensor nodes (video, audio and scalar) are used to collect the information.

ISSN (Print): 2204-0595

ISSN(Online):22031731 
The senor nodes are arranged for sensing and broadcasting the agriculture soil information like, leave still images, recorded sound of animals, birds, soil temperature, moisture, air quality and nutrients.
The configuration of the sensor node is shown in Figure 4. The information obtained is transmitted to the Gateway.

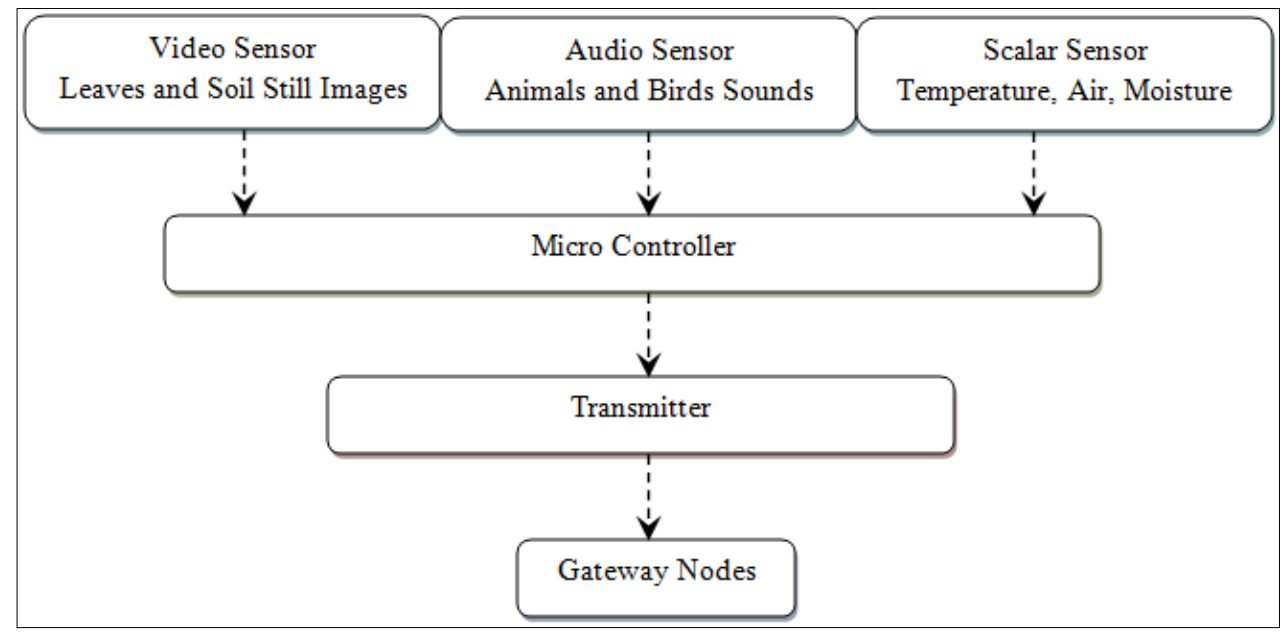

Figure 3 Structure of Sensor

Gateway Nodes: The gateway nodes are responsible for processing the sensed information from the sensor nodes and transmitting it to the Base
Station. Gateway nodes maintain the priority queues shown in Figure 4.

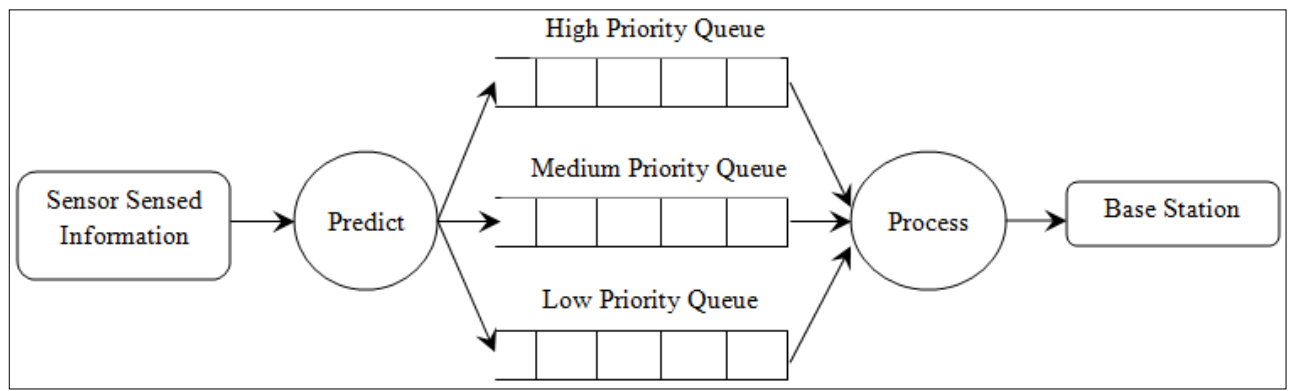

Figure 4 Priority Structure of Gateway Node

Base Station: The base station receives gateway information and transmits it via an internet link to the user. Base station collects the information about the agriculture soil and sends to user to take the corresponding action.

\subsection{Sleep Wake- up Model}

This section explains the sleep wakeup model of the proposed work. The network of sensing area is splits into number of regions. Each region contains certain number of sensor nodes. Randomly selects $50 \%$ of nodes in each region and set a time slot for sleep and wakeup.

A Sensor in sleep mode, it cannot sense or transmit the information. A tiny amount of energy is consumed when the node is in sleep mode. A sensor can sense and transmit the information to gateway during wakeup mode. This mode consumes more energy compared to the sleep mode.

\section{CONCLUSION}

Wireless multimedia sensor network is used in many different fields. In this paper, agriculture soil monitoring using WMSN. This method considers the priority aware QoS based energy efficient data transmission for WMSN. Compared to the other current systems, this approach decreases the energy usage of the network. Agriculture soil information is used for predict the soil nutrients, leaves disease analysis and monitor the agriculture environments. 
The end user can use this valid information to take the further decision.

\section{Reference}

[1] I.T. Almalkawi, M.G. Zapata, J.N. Al-Karaki, J. Morillo-Pozo, (2010) "Wireless Multimedia Sensor Networks: Current Trends and Future Directions", Sensors (Basel), vol. 10, no. 7, pp. 6662-6717

[2] I.F.Akyildiz, T. Melodia, K.R.Chowdhury, (2007) "A survey on wireless multimedia sensor networks", Computer Networks, vol. 51, no. 4, pp. 921-960

[3] H. Shen, and G. Bai, (2016) "Routing in wireless multimedia sensor networks: A survey and challenges ahead", Journal of Network and Computer Applications, vol. 71, pp. 30-49.

[4] M.N. Hayat, H. Khan, Z. Iqbal, Z. Ur Rahman and M. Tahir, (2017) "Multimedia sensor networks: Recent trends, research challenges and future directions," 2017 International Conference on Communication, Computing and Digital Systems (C-CODE), pp. 157-162.

[5] A. Ali, Y. Ming, S. Chakraborty and S. Iram, (2017), "A Comprehensive Survey on Real-Time Applications of WSN ", Future Internet, vol. 9, no. 4.

[6] J. John1, G.S. Kasbekar, D.K. Sharma, V. Ramulu, M.S. Baghini, (2018), "Design and Implementation of a Wireless SensorNetwork for Agricultural Application", EAI Endorsed Transactions on Internet of Things, vol. 4, no. 16.

[7] A. Genta, D.K. Lobiyal, and J.H. Abawajy, (2019), "Energy Efficient Multipath Routing Algorithm for Wireless Multimedia Sensor Network", Sensors, vol. 19, no. 17.

[8] X. Mao, X. Miao, Y. He, X. Li and Y. Liu, (2012) "CitySee: Urban CO2 monitoring with sensors," 2012 Proceedings IEEE INFOCOM, pp. 1611-1619.

[9] D. Hasenfratz, O. Saukh, C. Walser, C. Hueglin, M. Fierz, T. Arn, J. Beutel, L. Thiele, (2015) "Deriving high-resolution urban air pollution maps using mobile sensor nodes" ,Pervasive and Mobile Computing, vol. 16, pp. 268-285
[10] Q. Jiang, F. Kresin, A. Bregt, L. Kooistra, E. Pareschi, E. van Putten, H. Volten, J. Wesseling, (2016) "Citizen Sensing for Improved Urban Environmental Monitoring", Journel of Sensors.

[11] R. Pahuja, H.K. Verma and M. Uddin, (2013) "A Wireless Sensor Network for Greenhouse Climate Control," in IEEE Pervasive Computing, vol. 12 , no. 2 , pp. $49-58$

[12] J. Liu, (2016), "Design and Implementation of an Intelligent Environmental-Control System: Perception, Network, and Application with Fused Data Collected from Multiple Sensors in a Greenhouse at Jiangsu, China", International Journal of Distributed Sensor Networks, vol. 12, no. 7

[13] H.M. Abdulsalam, B.A. Ali and A. Alyatama (2016), "Air Quality Monitoring Using a LEACHbased Data Aggregation Technique in Wireless Sensor Network", Adhoc \& Sensor Wireless Networks, vol. 32 no. 3, pp. 275-300.

[14] H. Shi, K.M. Hou, X. Diao, X. Liu, J.J. Li, C. de Vaulx, (2013), "A wireless multimedia sensor network platform for environmental event detection dedicated to precision agriculture", New and Smart Information Communication Science and Technology to Support Sustainable Development: Workshop France-Chine (NICST 2013), 2013.

[15] K.N.Bhanu, T. Bhaskara Reddy and M.Hanumanthappa (2019), "Multi-agent based context aware information gathering for agriculture using Wireless Multimedia Sensor Networks", Egyptian Informatics Journal, vol. 20, no. 1, pp. 3344

[16] W. Bi (2019), "Design of Intelligent Monitoring Architecture for Multimedia Sensor Networks in Crop Areas", agronomia journal, Vol. 36, No. 6

[17] K. Indumathi, R. Hemalatha, S. A. Nandhini and S. Radha, (2017) "Intelligent plant disease detection system using wireless multimedia sensor networks," 2017 International Conference on Wireless Communications, Signal Processing and Networking (WiSPNET), pp. 1607-1611.

[18] Z. Libo, H. Tian and Guan Chunyun, (2019) "Wireless multimedia sensor network for rape disease detections", Journal on Wireless Communications and Networking, 
[19] A. Bouchemel, D. Abed and A. Moussaoui, (2018) "Enhancement of Compressed Image Transmission in WMSNs Using Modified \$ $\backslash$ mu\$ Nonlinear Transformation," in IEEE Communications Letters, vol. 22, no. 5, pp. 934-937

[20] M.A. Habib and S. Moh,(2019) "Robust Evolutionary-Game-Based Routing for Wireless Multimedia Sensor Networks", Sensors, vol. 19, no. 16.

[21] M.Z. Hasan, F. Al-Turjman and H. Al-Rizzo, (2017) "Optimized Multi-Constrained Quality-ofService Multipath Routing Approach for Multimedia Sensor Networks," in IEEE Sensors Journal, vol. 17, no. 7, pp. 2298-2309

[22] W. Zhang, Y. Liu, G. Han, Y. Feng and Y. Zhao, (2018) "An Energy Efficient and QoS Aware Routing Algorithm Based on Data Classification for Industrial Wireless Sensor Networks," in IEEE Access, vol. 6, pp. 46495-46504. 